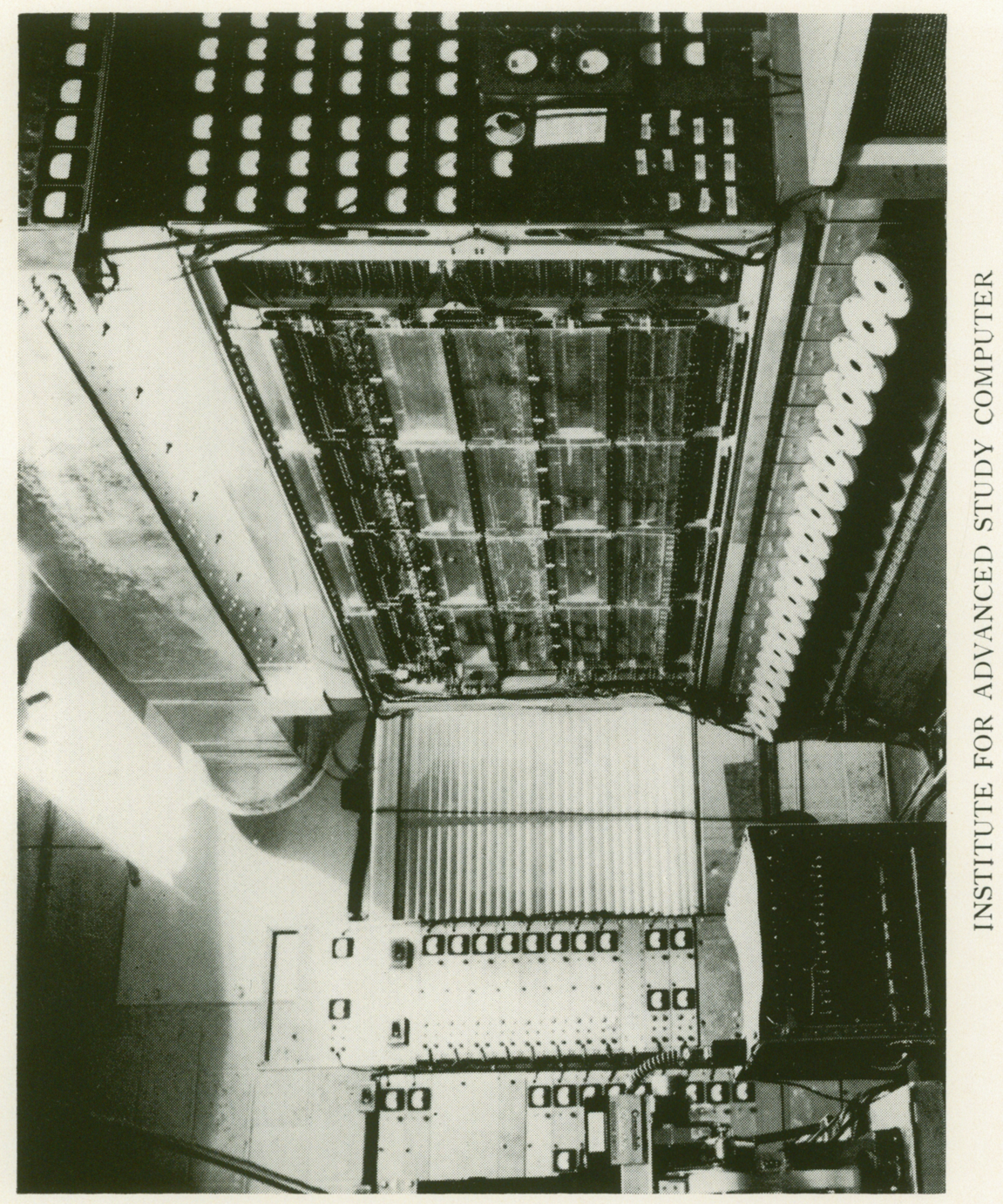




\section{A Technique for Real Time Simulation of a Rigid Body Problem}

1. Introduction. With the advent of large-scale high-speed digital computers, there arises the question of their possible use in the solution of problems in "real time," i.e., in conjunction with instruments receiving and responding to stimuli from the external environment. The criteria for satisfactory operation in such real-time service are different from those generally encountered. First, the computer must calculate fast enough to respond to the fastest changing stimuli; speed in solving the associated equations is in fact the primary requirement. Again, because it is impossible accurately to predict stimuli variations, either the response must be delayed or a "reasonable" amount of inaccuracy must be tolerated or both. Third, because of the limited range of number representation in the digital computer (or, for that matter, in any computer), all parameters must remain bounded no matter when or where they occur. Finally, the computations must be stable.

This paper is devoted to a discussion of the manipulation of the dynamical equations of a rotating rigid body with fixed center of gravity. ${ }^{1}$ The techniques here adapted will be seen to sacrifice accuracy to speed of computation, but in such a way as to hold inaccuracies reasonably low; at the same time, a singularity generally encountered will be circumvented. Stability will not be discussed.

2. Manipulation of Eulerian angles. The equations of motion of a rotating rigid body are usually simplified by using a set of axes attached to the rigid body. The simplification results from the fact that this choice of axes causes the moments and products of inertia to become constants. In addition, the products of inertia can be made to vanish by properly orienting these axes with respect to the rigid body.

The orientation of the rotating axes $O X, O Y$, and $O Z$ relative to a set of fixed axes $O X_{0}, O Y_{0}$, and $O Z_{0}$ may be defined by the Eulerian angles $\psi, \theta, \phi$. For convenience assume $O Z_{0}$ downward, $O X_{0}$ North, and $O Y_{0}$ East. $\psi$ is the angle measured about $O Z_{0}$ from the $X_{0} Z_{0}$ plane to the (vertical) plane containing $O Z_{0}$ and $O X$; it is positive when $O X$ is toward the East. $\theta$ is the angle between $O X$ and the $X_{0} Y_{0}$ plane; it is positive when $O X$ is above the $X_{0} Y_{0}$ plane. It is measured about an axis in the $X_{0} Y_{0}$ plane. Call this axis $O Y^{\prime} . \phi$ is the angle measured about the $O X$ axis from $O Y^{\prime}$ to $O Y$; it is positive for clockwise rotation as viewed from the origin along the positive $O X$ axis.

If the angular velocity of the moving axes has components $p, q$, and $r$ about the moving axes $O X, O Y$, and $O Z$, respectively, then the following three differential equations show the relations among the angles and the components of angular velocity.

$$
\begin{aligned}
\dot{\theta} & =q \cos \phi-r \sin \phi, \\
\dot{\phi} & =\frac{1}{\cos \theta}(p \cos \theta+q \sin \theta \sin \phi+r \sin \theta \cos \phi), \\
\psi & =\frac{1}{\cos \theta}(q \sin \phi+r \cos \phi) .
\end{aligned}
$$


The presence of $\cos \theta$ in the denominator makes two of these equations indeterminate when $\theta$ is $\pm 90^{\circ}$. This makes the equations difficult to use especially where point by point numerical computations are being performed. One common method is to limit the variable $\theta$ say to the range $\pm 85^{\circ}$. This is avoiding the problem rather than solving it.

This indeterminism is a product of the mathematical approach to a physical problem. A different choice of axes for example would present the same difficulty at a different orientation of the rotating body. Therefore the way out of the difficulty required a new mathematical point of view.

3. Derivation of direction cosine equations. When the equations of motion contain the Eulerian angles they appear in combinations equal to one or more of the direction cosines. That is, the Eulerian angles can be eliminated from the mechanical equations of motion by introducing the direction cosines as parameters. The kinematic differential equations for the direction cosines are quite simple and have no indeterminate points. This is the principal gain.

The new differential equations can be derived by the following method. The unit vector along the $X_{0}$ axis is related to the unit vectors along the moving $X, Y, Z$ axes by

$$
i_{0}=i l_{1}+j m_{1}+k n_{1} .
$$

Differentiate this with respect to time

$$
0=\frac{d i}{d t} l_{1}+i i_{1}+\frac{d j}{d t} m_{1}+j \dot{m}_{1}+\frac{d k}{d t} n_{1}+k \dot{n}_{1} .
$$

If the moving axes are rotating with an angular velocity $i p+j q+k r$

$$
\frac{d i}{d t}=j r-k q, \quad \frac{d j}{d t}=k p-i r, \quad \frac{d k}{d t}=i q-j p .
$$

Substituting these values for the derivatives we obtain

$$
i\left(\dot{l}_{1}-r m_{1}+q n_{1}\right)+j\left(\dot{m}_{1}-p n_{1}+r l_{1}\right)+k\left(\dot{n}_{1}-q l_{1}+p m_{1}\right)=0,
$$

which requires that

$$
\begin{aligned}
\dot{l}_{1} & =r m_{1}-q n_{1}, \\
\dot{m}_{1} & =p n_{1}-r l_{1}, \\
\dot{n}_{1} & =q l_{1}-p m_{1} .
\end{aligned}
$$

A similar treatment of $j_{0}$ and $k_{0}$ yields similar equations with subscripts 2 and 3 , respectively.

4. Identities. Since there were three independent differential equations involving the Eulerian angles only three of these nine new differential equations can be expected to be independent of the 21 identities that obtain for direction cosines. These 21 identities include six expressions for normality

$$
l_{1}{ }^{2}+m_{1}{ }^{2}+n_{1}{ }^{2}=1, \text { etc. }
$$

six for orthogonality

$$
l_{1} l_{2}+m_{1} m_{2}+n_{1} n_{2}=0, \text { etc. }
$$


and nine of the form

$$
l_{1}=m_{2} n_{3}-m_{3} n_{2}, \text { etc. }
$$

To choose three independent equations, choose equations all of which do not have the same subscripts nor do all involve the time derivative of the same letter. For example

$$
\begin{aligned}
i_{1} & =r m_{1}-q n_{1}, \\
\dot{l}_{2} & =r m_{2}-q n_{2}, \\
\dot{m}_{2} & =p n_{2}-r l_{2}
\end{aligned}
$$

is a set of independent equations which, with six of the identities, can be used to obtain all nine direction cosines. If desired, more than three differential equations and fewer than six identities can be used. For some applications, such as analogue computers, the differential equations are more suitable than the identities. Even for digital computers the differential equations may be preferred to identities of the form of equations (1) and (2).

5. Computation Procedure. Before discussing the plan of computation, it will be well to discuss a fundamental change that must often be made. Since there is no unity in many modern digital computers, this number cannot be held in the machine. Indeed, if a direction cosine were computed correctly to be unity, it would appear in such a machine as zero. To remove this difficulty, pseudo direction cosines can be introduced, which are $M$ times the corresponding true direction cosines. For example, with $M=1 / 2$, the identities are of the form

$$
\begin{aligned}
l_{3}^{2}+m_{3}^{2}+n_{3}{ }^{2}=\frac{1}{4} & \text { (6 of these), } \\
l_{3} l_{2}+m_{3} m_{2}+n_{3} n_{2}=0 & \text { (6 of these), } \\
l_{1}=2\left(m_{2} n_{3}-m_{3} n_{2}\right) & \text { (9 of these) }
\end{aligned}
$$

The first step in the proposed computation procedure is the use of three differential equations to compute $l_{3}, m_{3}, n_{3}$. Since both curtailment and round-off errors will gradually cause these parameters to fail to obey the normality equations, a second step is required to correct this failure.

Let the computed values be $l_{3}{ }^{\prime}, m_{3}{ }^{\prime}, n_{3}{ }^{\prime}$. Define $\epsilon$ by the equation:

$$
l_{3}{ }^{2}+m_{3}{ }^{2}+n_{3}{ }^{2}=M^{2}(1+\epsilon) .
$$

A normalized set of direction cosines is therefore

$$
l_{3}{ }^{2}=\frac{l_{3}{ }^{2}}{1+\epsilon}, \quad m_{3}{ }^{2}=\frac{m_{3}{ }^{2}}{1+\epsilon}, \quad n_{3}{ }^{2}=\frac{n_{3}{ }^{2}}{1+\epsilon} .
$$

By using two terms in the binomial expansion of $(1+\epsilon)^{-7}$,

$$
l_{3}=l_{3}{ }^{\prime}\left(1-\frac{\epsilon}{2}\right)=2 l_{3}{ }^{\prime}\left(\frac{1}{2}-\frac{\epsilon}{4}\right), \text { similarly } m_{3}, n_{3},
$$

thus avoiding the square root computation.

$l_{2}^{\prime}, m_{2}{ }^{\prime}, n_{2}{ }^{\prime}$ may now be computed from the appropriate three differential equations. Consider the two vectors,

$$
\begin{gathered}
r_{3}=i l_{3}+j m_{3}+k n_{3}, \\
r_{2}^{\prime}=i l_{2}^{\prime}+j m_{2}^{\prime}+k n_{2}^{\prime} .
\end{gathered}
$$


The vector $r_{3}$ has magnitude $M$. The vector $r_{2}^{\prime}$ should, but in general will not, have magnitude $M$ and be perpendicular to $r_{3}$. Let $r_{2}^{\prime}$ be turned through as small an angle as possible to make it perpendicular to $r_{3}$. To do this it is necessary only to look for $r_{2}$ in the plane of $r_{2}^{\prime}$ and $r_{3}$, i.e.,

$$
r_{2}=(1+a) r_{2}{ }^{\prime}+b r_{3} \text {. }
$$

The scalars $a$ and $b$ must at the same time make the magnitude of $r_{2}$ equal to $M$.

Define $\epsilon_{1}$ and $\epsilon_{2}$ by the equations

$$
\begin{aligned}
l_{2}{ }^{\prime 2}+m_{2}{ }^{2}+n_{2}{ }^{\prime 2} & =M^{2}\left(1+\epsilon_{1}\right), \\
l_{2}^{\prime} l_{3}+m_{2}^{\prime} m_{3}+n_{2}^{\prime} n_{3} & =M^{2} \epsilon_{2} .
\end{aligned}
$$

If the errors $\epsilon_{1}$ and $\epsilon_{2}$ are small, the corrections $a$ and $b$ will be small; then it will be sufficiently exact to neglect second degree terms in $a, b, \epsilon_{1}, \epsilon_{2}$. Requiring $r_{2}$ to have magnitude $M$ leads to

$$
a=-\frac{\epsilon_{1}}{2},
$$

while the fact that $r_{2}$ is normal to $r_{3}$ yields

$$
b=-\epsilon_{2} \text {. }
$$

Thus, neglecting second order terms, the corrected values may now be written

$$
\begin{aligned}
& l_{2}=l_{2}^{\prime}\left(1-\frac{\epsilon_{1}}{2}\right)-l_{3 \epsilon_{2},} \\
& m_{2}=m_{2}^{\prime}\left(1-\frac{\epsilon_{1}}{2}\right)-m_{3} \epsilon_{2}, \\
& n_{2}=n_{2}^{\prime}\left(1-\frac{\epsilon_{1}}{2}\right)-n_{3} \epsilon_{2} .
\end{aligned}
$$

The correction involving $\epsilon_{1}$ adjusts the magnitude while the correction involving $\epsilon_{2}$ corrects the direction of $r_{2}$. The results obtained show that to the first order either correction may be applied first or they may both be applied together.

There is a second method of orthogonalizing, which is preferable because it is quicker. In this method the computed values of two of $l_{2}, m_{2}, n_{2}$ are assumed correct and the third is adjusted to make $r_{2}$ perpendicular to $r_{3}$. If $n_{3}$ is greater than either $l_{3}$ or $m_{3}$, for example, only $l_{2}$ and $m_{2}$ are computed by integrating their differential equations; $n_{2}$ is then determined from the orthogonality condition

$$
n_{2}=\frac{l_{2} l_{3}+m_{2} m_{3}}{-n_{3}} .
$$

Note that if $n_{2}{ }^{\prime}$ had been computed from the differential equation, then

$$
\begin{gathered}
l_{2} l_{3}+m_{2} m_{3}+n_{2} n_{3}=\epsilon_{3} M^{2}, \\
n_{2}=\frac{n_{2} n_{3}-\epsilon_{3} M^{2}}{n_{3}}=n_{2}{ }^{\prime}-\frac{\epsilon_{3} M^{2}}{n_{3}} .
\end{gathered}
$$


This amounts to correcting $n_{2}{ }^{\prime}$ by the term

$$
-\frac{\epsilon_{3} M^{2}}{n_{3}}
$$

Obviously if $l_{2}{ }^{\prime}$ had been corrected instead of $n_{2}{ }^{\prime}$ the correction would be

$$
-\frac{\epsilon_{3} M^{2}}{l_{3}}
$$

To make the correction small, the largest of $l_{3}, m_{3}, n_{3}$ is made to appear in the denominator. This also prevents division by zero, which might occur if $n_{2}$ were corrected under all circumstances.

After the direction of $r_{2}$ has been established perpendicular to $r_{3}$, its magnitude must be adjusted. The procedure is to alter all components proportionately as for $r_{3}$ above. Clearly this does not destroy orthogonality. $l_{1}, m_{1}, n_{1}$ can now be computed from equation (3)

$$
l_{1}=\frac{m_{2} n_{3}-m_{3} n_{2}}{M}, \text { etc. }
$$

6. Conclusions. The equations of motion of a rotating rigid body can be formulated directly in terms of the direction cosines. The direction cosines can be determined as described above in a manner which is thought to be ideally suited to digital computation. No trigonometric functions of the Eulerian angles appear, obviating the necessity of referring to a function table or to a series expansion to obtain the solutions of the equations of motion. In addition, all quantities are finite for all orientations of the rigid body.

This work was done in connection with a study performed by the University of Pennsylvania, Moore School of Electrical Engineering, under Contract N6Onr 24913 sponsored by the Office of Naval Research, Special Devices Center, Port Washington, New York.

H. J. Gray, Jr.

M. RUBINOFF

H. SOHON

University of Pennsylvania

Moore School of Electrical Engineering

${ }^{1}$ LeIgh Page, Introduction to Theoretical Physics, Second Edition-Fourteenth Printing, D. Van Nostrand Company, Inc., New York, N. Y., Introduction, Chap. I, II; 1951.

\section{A Solution of Simultaneous Linear Equations and Matrix Inversion with High Speed Computing Devices}

In solving several systems of simultaneous linear equations, in which the coefficients of the unknowns are the same in all the systems but in 